EPJ Web of Conferences 32, 02016 (2012)

DOI: $10.1051 /$ epjconf/20123202016

(C) Owned by the authors, published by EDP Sciences, 2012

\title{
Studies on absorption of EC waves in assisted startup experiment on FTU
}

\author{
G. Granucci ${ }^{1}$, D.Ricci ${ }^{1}$, D. Farina ${ }^{1}$, L. Figini ${ }^{1}$, D.Iraji ${ }^{1}$, O.Tudisco ${ }^{2}$, G. Ramponi ${ }^{1}$ and W.Bin ${ }^{1}$ \\ ${ }^{1}$ Istituto di Fisica del Plasma, CNR, EURATOM-ENEA Association, Milan, Italy \\ ${ }^{2}$ EURATOM-ENEA Association, C.R. Frascati, Italy
}

\begin{abstract}
Assistance of EC wave for plasma breakdown and current ramp up is the proposed scenario for the ITER case, characterized by low toroidal electric field. The experimental results on many tokamaks clearly indicate the capabilities of the proposed scheme to have a robust breakdown in ITER. The key aspect of this technique is the EC power required, strongly related to the absorption of the wave in the initial stage of plasma formation. This aspect is generally neglected due to the diagnostics difficulties in the plasma formation phase. As a consequence a multi-pass absorption scheme is usually considered reasonable, leading to a strong absorption after many reflections on the walls. The present study exploits the high temporal and spatial resolution of the fast scanning interferometer of FTU together with the measure of residual power obtained by a sniffer probe. The absorbed EC power is calculated considering also the polarization rotation and the subsequent mode conversion after incidence on the internal wall and compared with that derived from experimental data. The resulting EC power distribution can explain differences observed between perpendicular and oblique injection results, indicating future investigations to define ITER power requirements.
\end{abstract}

\section{Introduction}

Studies on EC assisted startup scenarios are strongly motivated by preparation of ITER. It is well known that in ITER the toroidal electric field will be limited to $0.3 \mathrm{~V} / \mathrm{m}$ and that EC power will be required during breakdown. For these reasons a strong experimental activity has been done on many tokamaks (DIII-D, TS, FTU, AUG, K-STAR, T-10, JT-60-U) obtaining favourable results for ITER [1]. Nevertheless there are some aspects that needs more focused analysis, especially in the attempts to give a more consolidate prediction on EC power requirements. The ITER EC system is foreseen to inject power obliquely with toroidal angles in the range $20^{\circ}-40^{\circ}$ for the Equatorial Launcher and $18^{\circ}$ or $20^{\circ}$ (depending on the row) for the Upper Launcher [2]. A key aspect is a correct evaluation of the EC absorbed power and its localization, assuming that it is more effective to deposit it in the magnetic null. Absorption is generally assumed high, and total after several reflections at the wall [3] including a favourable polarization conversion (from OM to XM) occurring at wall reflection in case of oblique injection. Nevertheless no detailed experimental data or computational analysis, on power absorption in the initial phase of tokamak plasma, are available up to now. In this work a comparison of absorption between perpendicular and oblique injection is presented on the basis of FTU data [4], as a first attempt to explain the observed differences between the two cases and in view of an extrapolation to the ITER case.

This is an Open Access article distributed under the terms of the Creative Commons Attribution License 2.0, which permits unrestricted use, distribution, and reproduction in any medium, provided the original work is properly cited. 


\section{EC assisted startup experiments}

\subsection{FTU experimental setup}

The experimental results on which the present work is based have been obtained on FTU (Frascati Tokamak Upgrade) during a dedicated campaign to study EC assisted breakdown. FTU is a compact tokamak $\left(\mathrm{R}_{0}=0.935 \mathrm{~m}, \mathrm{a}=0.3 \mathrm{~m}\right)$ capable to operate in a wide toroidal field range $(2.5-8 \mathrm{~T})$ and equipped of an ECRH system [5] (4 tubes at $140 \mathrm{GHz}, 0.5 \mathrm{MW}$ each, pulsed for $0.5 \mathrm{~s}$ ) injecting the power beams from equatorial ports in the low field side. The waves are polarized in the horizontal plane, corresponding to ordinary mode $(\mathrm{OM})$ in case of perpendicular injection. A polarization mismatch of about $17 \%$ is expected for the oblique injection angle used in the experiment $\left(20^{\circ}\right)$.

EC assisted breakdown experiments [3] have been performed varying filling pressure, toroidal electric field and to start preliminary comparison between perpendicular and oblique injection of EC waves [4]. In the experiments the power was switched on before the start of the electric field, raised from zero to the set value. Two levels of power were used $(400 \mathrm{~kW}$ or $800 \mathrm{~kW})$ and part of the scan was done with oblique injection at $20^{\circ}$. Major results are the demonstration of a low level breakdown $(0.4 \mathrm{~V} / \mathrm{m}$ minimum that is more than 6 times less than the usual ohmic robust one), a pressure limit higher (4 time larger) for a sustained breakdown, and a moderate (16\%) flux saving. Behind these results, a general reduced effectiveness was observed in the case of the oblique injection. The results of a pressure scan, done at two levels of EC power, are shown in Figure 1.

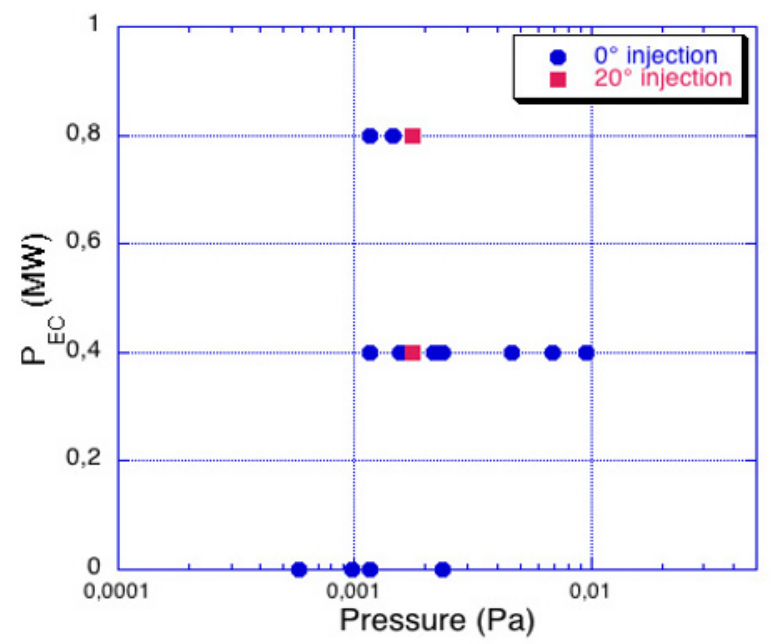

Fig. 1. Pressure scan from FTU EC assisted experiments. The red squares represent oblique injection. The high power case $(800 \mathrm{~kW})$ requires more experiments. For all the presented data, the toroidal electric field is $1.5 \mathrm{~V} / \mathrm{m}$.

The pressure limit for oblique injection seems to be similar to that of the ohmic case and is clearly lower than the one for perpendicular injection. Moreover, a reduced plasma current ramp-up has been observed: twice the power is required to obtain results similar to those of perpendicular injection [4]. On the other hand, oblique injection exhibits a wider and higher density profile at the power switch-on. It has to be mentioned that when oblique injection is used a second H-alpha peak is observed at the beginning of the current ramp, that is $\sim 50 \mathrm{~ms}$ after the beginning of loop voltage ramp. This observation has no clear explanation at present, and it will be object of further investigations. 


\subsection{Evidences of different power depositions}

In order to investigate the differences in effectiveness observed between oblique and perpendicular injection we have analyzed the power depositions in the two cases. A first observation was done using the bremsstrahlung emission as viewed by a vertical camera. The results are reported in Figure 2 where contour plots characterize the intensity versus time. Clearly the oblique injection case exhibits a larger emission profile, starting from the very beginning of the pulse (here EC power is switched on at $\mathrm{t}=0$, while the current starts to ramp $50 \mathrm{~ms}$ later). A similar behaviour is evidenced by density measurement, from SIRIO interferometer [6]: the density build up in case of oblique injection is faster, higher and wider, even from the beginning of gas ionization. A similar distribution is also observed using the video camera looking at the interior of vacuum vessel during the discharge. In the first recorded frame (with an uncertainty of $\pm 10 \mathrm{~ms}$ ) a bright spot is observed close to the region where the power beam crosses the vertical cylinder of EC resonance. Light is emitted from a full toroidal ring, centred in the vessel, indicating that a kind of confinement is acting still from the initial phase of the pulse, even before the formation of current channel.
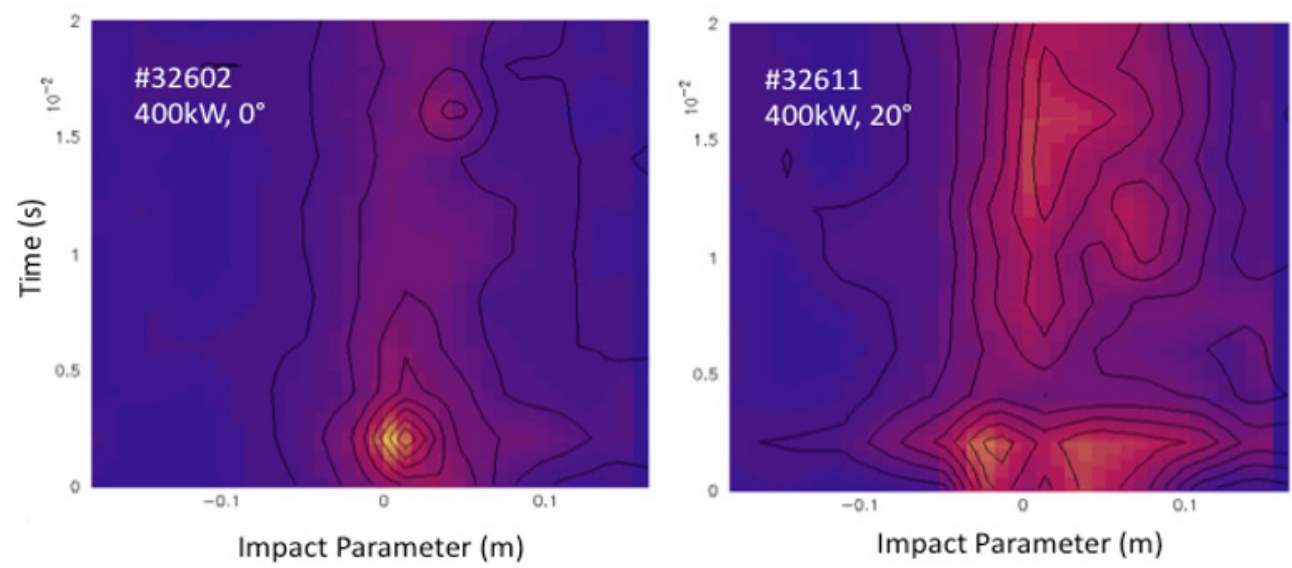

Fig. 2. Time evolution of contour plots (vs impact parameter) from bremsstrahlung vertical camera for perpendicular (left) and oblique (right) injection. $20^{\circ}$ case exhibits a wider plasma formation from the initial phase.

A comparison between perpendicular and oblique injection, done on the basis of the images recorded by the camera, confirms the more spread emitting profiles for $20^{\circ}$ toroidal injection. The intensity of the emission is at the same level in the two cases (as indicated by the vertical profile of visible light), but it is wider in the case of oblique injection.

\section{Analysis of EC absorption}

In order to verify the expected major absorption and explain the observed wider extension of the emitting profile in case of oblique injection, a comparison is made between the experimental data of not absorbed power obtained from a sniffer probe $120^{\circ}$ toroidally displaced with respect to launcher, and the absorbed power calculated by the analytical expressions for the EC optical depth [7] taking into account mode conversion at the inner wall.

\subsection{Mode conversion at the wall}

Considering the low temperature $(\sim 200 \mathrm{eV})$ and density $\left(10^{18}-10^{19} \mathrm{~m}^{-3}\right)$ of plasma in the early stage of the discharge, only part of the EC power is absorbed at first pass. Estimations based on FTU data have shown an absorption ranging from $2 \%$ to $10 \%$, depending on plasma density and electron 
temperature. The transmitted power is reflected by the inner wall if the toroidal injection angle is lower than the critical value $\sim 26.5^{\circ}$ (satisfied in the experiments), and then partially converted at the wall to extraordinary mode (XM). Here, wall reflection has been modelled assuming pure specular reflection from a metallic wall. The reflection at the wall has been considered ideal (no losses), while the metallic surface has been assumed to be a cylinder with axis on the tokamak axis. The fraction of EC power as OM after reflection is reported in Figure 3, as computed by the GRAY code [8] in which the polarization rotation has been included.

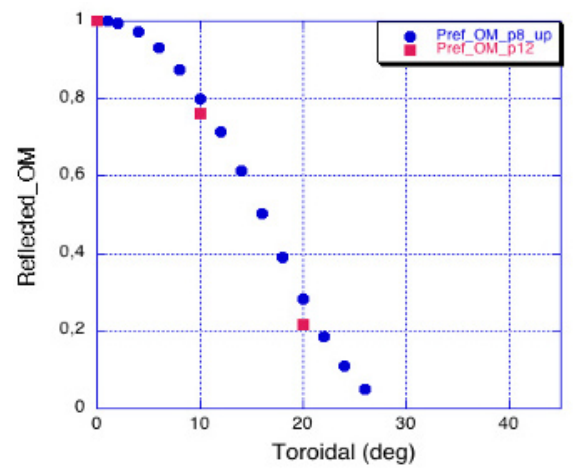

Fig. 3. Fraction of OM polarization after one reflection on inner wall of FTU. Red squares refers to equatorial launcher, blue circles to new upper launcher.

Power conversion into the more favourable XM yields a large increase in the absorption $(\sim 20$ times larger) but wider deposition distribution with respect to OM. This is due to the asymmetry of the absorption around the resonance for the XM: the wave propagating from HFS (after the reflection) starts to be absorbed before reaching the resonance position, and this results in a spread of the power in the radial direction.

\subsection{Absorption comparison}

The absorbed power is calculated considering two passes for both cases (oblique and perpendicular injection), by assuming that in the $20^{\circ}$ case the strong absorption of the converted XM makes negligible a possible third pass, and that in the $0^{\circ}$ case the transmitted power enters in the port aperture and can be considered absorbed after several reflections (the rate between port aperture and launcher internal surface is $\sim 1 / 350$ ). In the calculations the line integrated density from the fast scanning interferometer taken at the $\mathrm{EC}$ resonance $\left(\mathrm{R}_{\mathrm{EC}}=0.991 \mathrm{~m}\right)$ has been used, together with Michelson profile at $\mathrm{R}=\mathrm{R}_{\mathrm{EC}}$ for $\mathrm{T}_{\mathrm{e}}$, except when the data are not elaborated (due to the low signal/noise ratio) in which case a figure of $100 \mathrm{eV}$ has been considered. The results of the simulation are compared with the experimental data from sniffer probe and shown in Fig. 4 and Fig.5. The measured absorption and the calculated one are comparable in both cases. The observed discrepancies may be related to the normalization of the sniffer signal and to the reflection model on the inner toroidal limiter of FTU, assumed ideal in the calculations for the mode conversion.

To study the deposition of EC power, runs of GRAY code have been performed for the two pulses at a time with similar temperature and density $(\sim 70 \mathrm{~ms})$. The results are compared in Figure 6. For perpendicular injection the depositions occurs at approximately the same radial location for both first and second pass, with a total absorption of $21 \%$ and a peak of power density of $\sim 4 \mathrm{MW} \mathrm{m}^{-3}$. In the case of oblique injection mode conversion into XM at the inner reflection leads to an overall absorption of $85 \%$, but the power deposition is spread over a wide region, corresponding to $\sim 30 \%$ of the minor radius, with a peak of $3 \mathrm{MW} / \mathrm{m}^{3}$. 


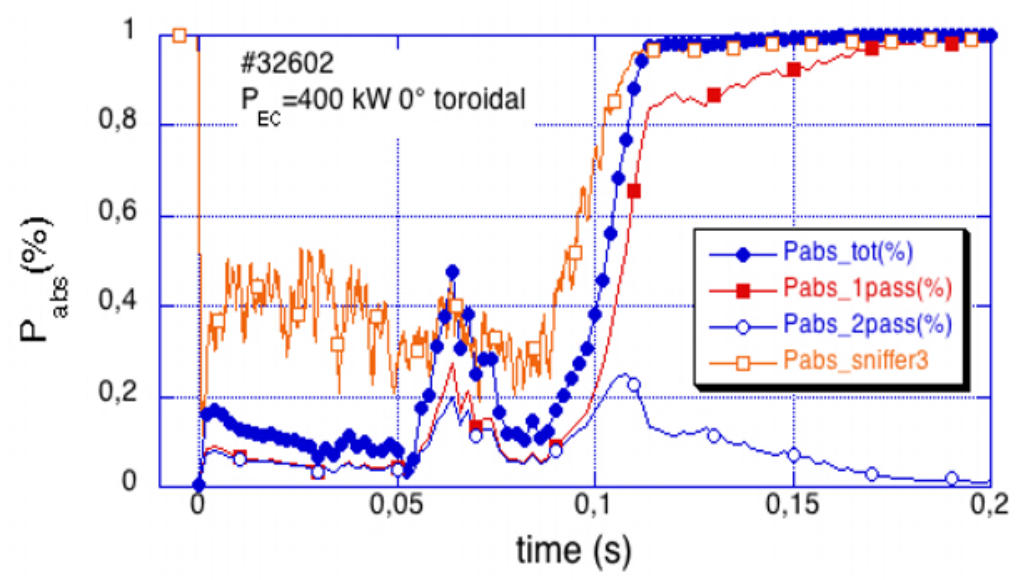

Fig. 4. Absorptions evolution for $0^{\circ}$ toroidal injection measured by sniffer probe signal (orange) and as calculated on the basis of the analytical expressions of the optical depths and considering two passes.

In fact, in case of propagation from the HFS XM absorption may occur before reaching the EC resonance, due to Doppler shift. This effect increases with Te and is responsible of the calculated power deposition profile that confirms experimental observations reported in Sec. 2. It has to be considered that the null field of FTU is $\sim 20 \mathrm{~cm}$ wide $(\sim 0.1 \mathrm{r} / \mathrm{a})$ starting from the inner side of the vessel and moving outward during the first tents of ms.

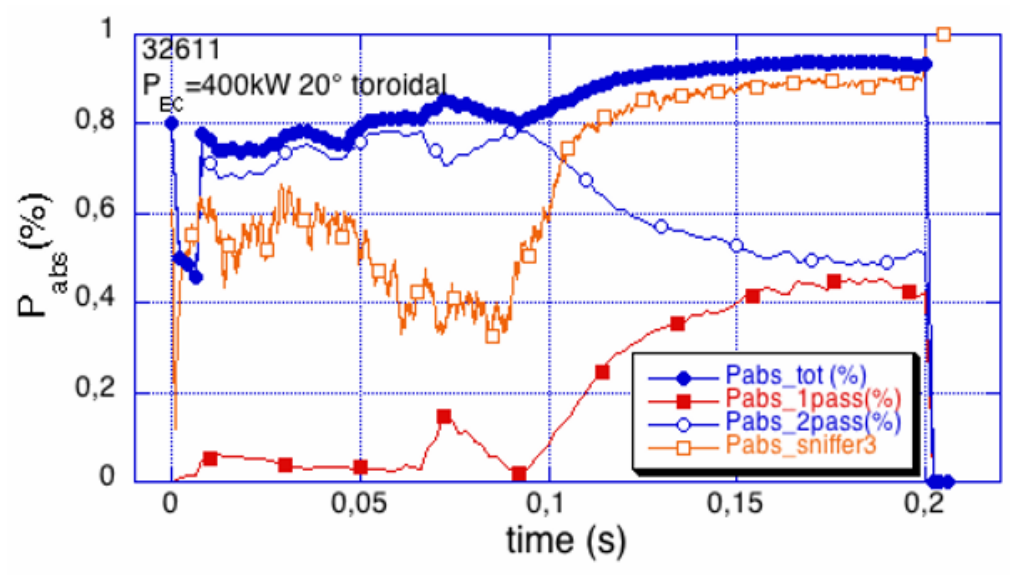

Fig. 5. Absorptions evolution for $20^{\circ}$ toroidal a conversion from $\mathrm{OM}$ to $\mathrm{XM}$ of $80 \%$ at reflection is considered.

Therefore it seems reasonable that, in case of oblique injection, part of the absorbed power falls in the region with shorter connection lengths (i.e. in the $\mathrm{Bz}>10 \mathrm{G}$ region) where electrons are quickly lost on the walls. The poor localization of oblique power absorption can be considered the reason of the reduced efficiency observed in sustaining breakdown, although an opposite behavior might be expected on the basis of the large amount of absorbed power. 

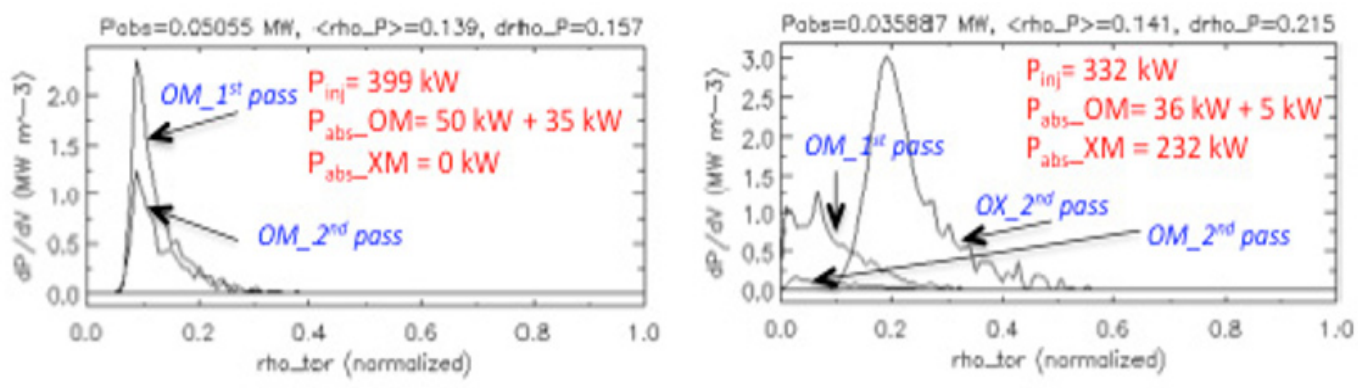

Fig. 6. Comparison of power deposition calculated with GRAY code at $70 \mathrm{~ms}$ after EC power switch on for the reference shots: \#32602 (left) and \#32611 (right). Note that in the case of perpendicular injection no conversion from OM to XM takes place at the wall reflection

\section{Conclusions}

The FTU experimental data, collected in the 2009 campaign dedicated to study EC assisted breakdown in a scenario similar to that foreseen in ITER, have put in evidence a reduced effectiveness of oblique injection (at $20^{\circ}$ ) with respect to perpendicular injection. To explain this observation, the EC power absorption has been investigated especially at the beginning of the plasma formation. The comparison is based on experimental data from sniffer probe (measuring the stray in the vessel) and the power absorption calculated [7] from experimental data ( $T_{e}$ and $n_{e}$, mainly), considering two passes through the resonant region. The mode conversion (from $\mathrm{OM}$ to $\mathrm{XM}$ ) at reflection on the inner wall of FTU has been estimated with the GRAY code, and found to be $\sim 80 \%$ for the used $20^{\circ}$ injection. The agreement between the calculations and the sniffer probe data is noticeable, showing a larger power absorption in case of the oblique injection with respect to the perpendicular injection, even for the initial plasma when the perpendicular one is still low (20\%). From the calculation of power deposition it is found that the oblique injection has a wider deposition profile (factor 2-3) with a peak lower than that obtained in the case of perpendicular injection. The power deposition seems to play therefore a crucial role for these two aspects: i) the peak value (i.e. power per particle) and ii) the localization (i.e. power absorbed within the field null). These two issues should be considered in defining the required EC power needed for ITER (an extensive beam tracing investigation has not yet been performed, especially after wall reflection). The preliminary analysis and results presented here should be confirmed and further investigated in the next experiments on FTU with the aid of the new fast steerable launcher [9] that allows a continuous control of toroidal injection angle.

\section{References}

1. J. Stober et al., Nucl. Fusion 51, 083031 (2011)

2. T. Omori et al., Fusion Eng. Des., 86951 (2011)

3. B. Lloyd et al., Plasma Phys. Controlled Fusion 38, 1627 (1996)

4. G. Granucci et al., Nucl. Fusion 51, 073042 (2011)

5. M. Aquilini et al., Fusion Science and Technology 45, 459 (2004)

6. A. Canton. et al., Applied Optics 45 (2006) 9105

7. Bornatici et al., Nucl. Fusion 23 (1983)

8. D. Farina, Fusion Science and Technology 52, 154 (2007)

9. A. Moro et al., this conference 